\title{
Experimental Study on the Constant Deceleration Scheme in Traveling Wave Direct Energy Converter ${ }^{*)}$
}

\author{
Yusuke TOGO, Tomohiro KATSURA, Hiromasa TAKENO, Yasuyoshi YASAKA, \\ Kazuya ICHIMURA $^{1)}$ and Yousuke NAKASHIMA ${ }^{1)}$ \\ Department of Electrical and Electronic Engineering, Kobe University, \\ 1-1 Rokkodai-cho, Nada-ku, Kobe 657-8501, Japan \\ 1) Plasma Research Center, University of Tsukuba, 1-1-1 Tennodai-cho, Tsukuba 305-8572, Japan
}

(Received 25 November 2014 / Accepted 25 January 2015)

\begin{abstract}
Traveling Wave Direct Energy Converter (TWDEC) was proposed to apply to D- ${ }^{3} \mathrm{He}$ nuclear fusion power generation system, and is studied as an energy source of a spacecraft recently where miniaturization of the device is important. Not only the modulation process, but also the deceleration process are important to treat a trade-off between device size and efficiency. The suitable scheme of the constant deceleration, of which experimental investigation was insufficient, was studied. Based on the theory of the scheme, a decelerator for simulation experiments was assembled and deceleration experiments were performed. The efficiency of the highest record in simulation experiments and a fair agreement with the theory were obtained. The problem was also clarified that the improvement of the modulation process was necessary.
\end{abstract}

(c) 2015 The Japan Society of Plasma Science and Nuclear Fusion Research

Keywords: advanced fusion, direct energy conversion, TWDEC

DOI: $10.1585 /$ pfr. 10.3405013

\section{Introduction}

In nuclear fusion power generation by $\mathrm{D}-{ }^{3} \mathrm{He}$ reaction, no high energy neutrons are produced and direct energy conversion (DEC) can be applied. The proposed DEC system is composed of a cusp-type direct energy converter (CuspDEC) and a traveling wave direct energy converter (TWDEC) [1]. The CuspDEC is placed in the front stage where thermal ions and electrons are discriminated and their energies are converted by conventional electrostatic converters. High energy protons pass through the CuspDEC and are led to the TWDEC placed in the latter stage. The kinetic energy of proton is converted into electricity by a realizable voltage.

Recently, the TWDEC is studied as an energy source of a spacecraft [2]. For usage in space, miniaturization of the device is important. Generally, large size is required to achieve high efficiency, and there is a trade-off between device size and efficiency in TWDEC. TWDEC is composed of a modulator and a decelerator. The incident protons are velocity modulated in the modulator and density modulated (bunched) in the downstream where the decelerator is settled. The bunching length is dominant in the axial size of the device [3], and a high modulation voltage is required for short bunching length, while it causes large velocity deviation of protons which results in low conversion efficiency.
In order to obtain high efficiency in the condition of short bunching length, the decelerator applicable to particles with large velocity deviation is needed. In the previous experimental studies on TWDEC, the optimum deceleration scheme was employed $[4,5]$. In this scheme, the structure of the traveling wave is matched to the particle with the standard velocity, and variation of velocity deviation cannot be treated. On one hand, the constant deceleration scheme was proposed theoretically [6], in which adjustment of deceleration voltage enabled variation of region trapping particles in phase space. The decelerator designed with the constant deceleration scheme has a possibility to miniaturize TWDEC with high conversion efficiency.

In this research, we examine the constant deceleration scheme experimentally as a first step for miniaturized TWDEC with high efficiency. Based on the theory, we design the decelerator for a TWDEC simulator, and perform deceleration experiment. Through the comparison with numerical calculation, the constant deceleration scheme is evaluated.

In Sec. 2, the experimental setup is explained with dividing into the experimental device, the theory of the constant deceleration scheme, and the design of the decelerator. In Sec. 3, the experimental results are shown, and discussion with a numerical result is also presented. In Sec. 4 , conclusion of the paper is given.

\footnotetext{
author'se-mail: takeno@eedept.kobe-u.ac.jp

*) This article is based on the presentation at the 24th International Toki Conference (ITC24).
} 


\section{Experimental Setup}

\subsection{Experimental device}

A Schematic view of the experimental device is shown in Fig. 1. The device consists of sections of an ion source, an accelerator, a modulator, a decelerator, and measurement.

In the ion source section, helium gas is supplied and the plasma is generated by an application of a magnetic field and a radio frequency (RF) power modulated by continuous pulses. In the accelerator section, ions are extracted by applying $V_{\text {ex }}$ to the electrode $\mathrm{P}$ and the electrode $\mathrm{E}$ is grounded. In addition, a cylindrical focusing electrode applying $V_{\mathrm{c}}$ is installed downstream of the extractor to suppress the diffusion of the beam.

The modulator is composed of four grid electrodes denoted $\mathrm{M}_{i}(i=1, \ldots, 4)$ (numbered in order in the direction of particle flow). All intervals between electrodes are $\lambda / 4$ where $\lambda$ is the wavelength of the traveling wave. The RF voltage of $V_{\text {mod }}$, the frequency of which is $7 \mathrm{MHz}$, is applied to the electrode $\mathbf{M}_{2}$, while the electrodes $\mathbf{M}_{1}, \mathbf{M}_{3}$, and $\mathrm{M}_{4}$ are grounded. In this condition, standing wave field (SWF) is created in the region between $M_{1}$ and $M_{2}$ and that between $\mathrm{M}_{2}$ and $\mathrm{M}_{3}$.

In the decelerator of the actual device, deceleration field is induced by incident bunched particles. In the simulation experiments, however, the induced field is too weak to decelerate as the beam current is small, so the deceleration field is excited by applying deceleration voltage $\left(V_{\mathrm{dec}}\right)$ externally. This scheme was called active decelerator [7] used in the previous experiments. $V_{\mathrm{dec}}$ is supplied by an RF source synchronized with $V_{\text {mod }}$.

In the measurement section, a Faraday cup is installed which is composed of an ion repeller grid (IR), a secondary electron repeller grid (SER), and a collector electrode (C). The ions are discriminated by IR applying sweeping voltage $V_{\text {ret }}$. The collector current $I_{\mathrm{c}}$ is averaged by a boxcar integrator synchronized by plasma production RF pulses, and recorded with swept $V_{\text {ret }}$. From the obtained characteristic of $I_{\mathrm{c}}\left(V_{\text {ret }}\right)$, an energy distribution of ion beam $f(E)$ can be derived from the following formula:

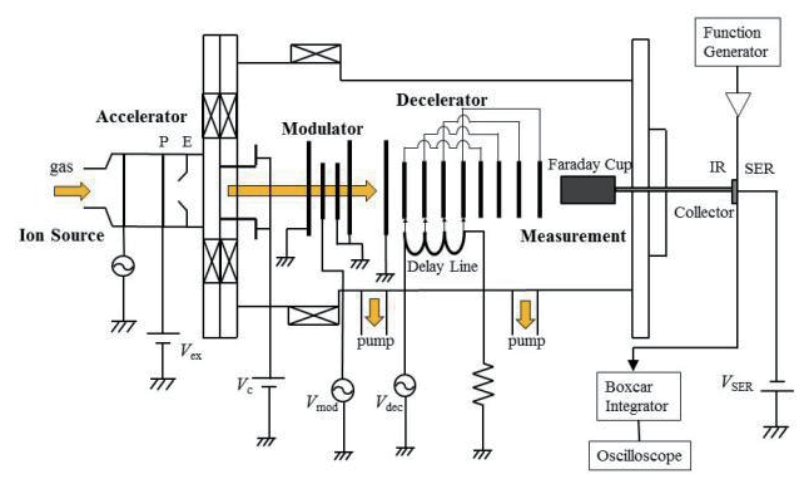

Fig. 1 Schematic view of the experimental device.

$$
f(E)=\left.\frac{1}{\sqrt{V_{\text {ret }}}} \frac{\mathrm{d} I_{\mathrm{c}}}{\mathrm{d} V_{\text {ret }}}\right|_{V_{\text {ret }}=E / e},
$$

where $E$ and $e$ are ion energy and unit charge, respectively.

\subsection{Principle of constant deceleration scheme}

In this section, the scheme of constant deceleration is briefly reviewed [6]. In the scheme, traveling wave with constant deceleration is created, and a part of the incident particles are trapped in the potential valley of the traveling wave. They make a bouncing motion in the potential valley, and are decelerated with the traveling wave.

The trapped condition of the particle can be understood by relative motion of the particle to the traveling wave. In Fig. 2, a trapped particle in a potential valley of the traveling wave is illustrated, where $s, u$, and $\Psi_{\mathrm{D}}$ are normalized position, velocity, and potential in the decelerator, respectively, the detail of which is described in Ref. 6. At the standing point in Fig. 2, which is expressed by phase angle $\theta_{\mathrm{s}}$, the inertia and the potential force are balanced. The particle make a bouncing motion between $\delta s_{1}$ and $\delta s_{2}$. If $\Psi_{\mathrm{D}}$ is small or the energy of the particle is large, motion of the particle is beyond the potential hill, and the particle is not trapped. It is more advantageous that the standing point is around the bottom of the valley, but it is limited by the relation:

$$
\alpha=\frac{2 \pi}{u_{\mathrm{ph}}} \Psi_{\mathrm{Dm}} \sin \theta_{\mathrm{s}},
$$

where $\alpha, u_{\mathrm{ph}}$, and $\Psi_{\mathrm{Dm}}$ are deceleration, phase velocity of the wave, and a maximum value of $\Psi_{\mathrm{D}}$, respectively. If we take a small value of $\theta_{\mathrm{s}}$, small $\alpha$ is necessary, which means decelerator length must be long.

The trapping region in a phase space is obtained by the following equation.

$$
\begin{aligned}
& \frac{\delta u}{\sqrt{\Psi_{\mathrm{Dm}}}}= \pm \sqrt{2 S} \\
& S=\cos \left(\frac{2 \pi}{u_{\mathrm{ph}}}\right) \delta s+\cos \theta_{\mathrm{s}}+\left(\frac{2 \pi}{u_{\mathrm{ph}}} \delta s+\theta_{\mathrm{s}}-\pi\right) \sin \theta_{\mathrm{s}}
\end{aligned}
$$

The region of Eq. (3) is indicated as the inside of the curve

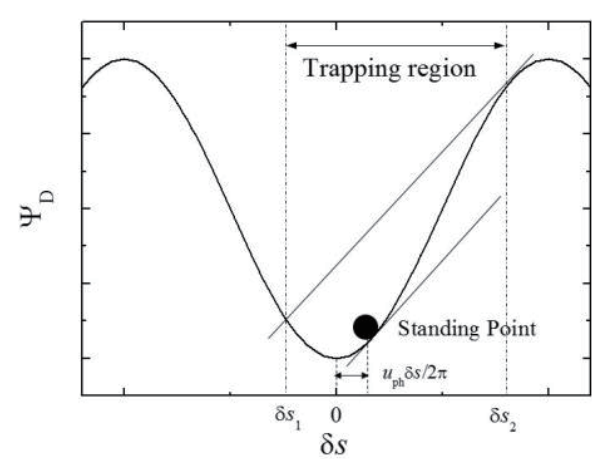

Fig. 2 A trapped particle in a potential valley. 


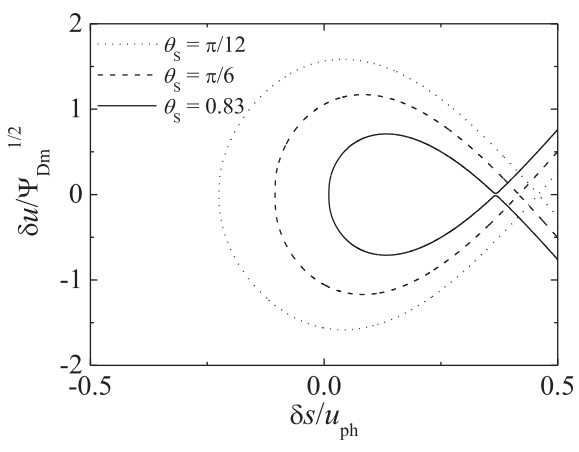

Fig. 3 Trapping region in the phase space.

Table 1 Installation position of decelerator electrodes.

\begin{tabular}{ccccccccc}
\hline Electrode & $\mathrm{D}_{1}$ & $\mathrm{D}_{2}$ & $\mathrm{D}_{3}$ & $\mathrm{D}_{4}$ & $\mathrm{D}_{5}$ & $\mathrm{D}_{6}$ & $\mathrm{D}_{7}$ & $\mathrm{D}_{8}$ \\
position [mm] & 0 & 14 & 26 & 38 & 48 & 57 & 66 & 73 \\
\hline
\end{tabular}

in Fig. 3, where three kinds of standing points are shown. As $\theta_{\mathrm{s}}$ increases, the trapping region becomes narrow.

\subsection{Design of the decelerator}

In a design of the decelerator for simulation experiments, energy reduction around $50 \%$ is taken as a goal within various limitations of the used devices. The incident helium ion energy is taken to be $3.2 \mathrm{keV}$ and the length of the decelerator of $3.5 \pi$ phase length with 8 electrodes is less than $0.1 \mathrm{~m}$, thus deceleration $\alpha$ is taken to be $8 \times 10^{11} \mathrm{~m} / \mathrm{s}^{2}$. When deceleration voltage $V_{\mathrm{dec}}=400 \mathrm{~V}_{0 \mathrm{p}}$, $\left(\mathrm{V}_{0 \mathrm{p}}\right.$ : zero-to-peak voltage) the phase of the standing point $\theta_{\mathrm{s}}=0.83$. The corresponding trapping region is shown in Fig. 3.

The electrode system consists of 8 grid electrodes denoted $\mathrm{D}_{i}(i=1,2, \ldots, 8)$ (numbered in order in the direction of particle flow) and grounded grid electrodes at the upstream of $\mathrm{D}_{1}$ and the downstream of $\mathrm{D}_{8} . V_{\mathrm{dec}}$ is applied to the electrodes $\mathrm{D}_{i}$ by a coaxial delay line on which are terminals to connect the electrodes at every $\pi / 2$ phase positions. The axial positions of $\mathrm{D}_{i}$ are taken to be at every $\pi / 2$ phases of the decelerating traveling wave, and determined as shown in Table 1.

The electrode system and the coaxial cable are assembled and calibrated. The electrodes $\mathrm{D}_{l}(l=5,6,7,8)$ are connected to $\mathrm{D}_{k}(k=1,2,3,4)$, respectively, as the phases of connected pair of electrodes are the same. Because of space capacitance of electrodes, the amplitudes and phases of $V_{\mathrm{dec}}$ have some errors to the ideal values as shown in Fig. 4. The errors of the phase are not so large, but those of amplitude are about $20 \%$. This electrode system is settled in the downstream of the modulator as $\mathrm{D}_{1}$ locates at the bunching position which is determined by numerical calculation with $V_{\text {mod }}=400 \mathrm{~V}_{0 \mathrm{p}}$.

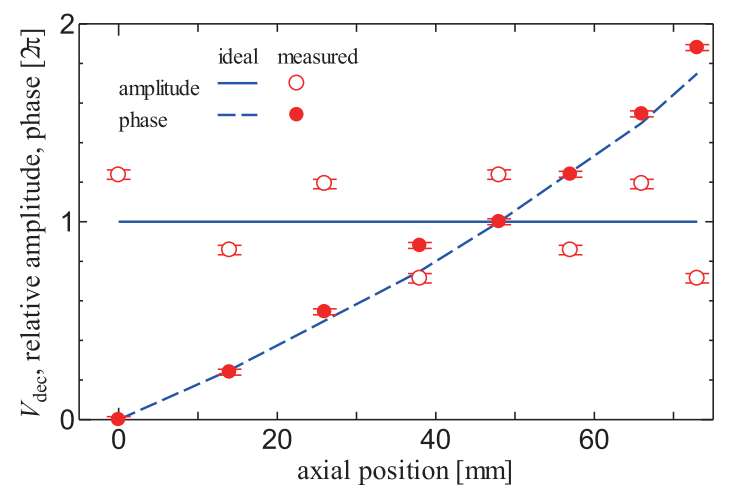

Fig. 4 Calibration of decelerator voltage.

\section{Experimental Result and Discus- sion}

The deceleration experiment was performed under the conditions of $V_{\mathrm{ex}}=3.2 \mathrm{kV}, V_{\mathrm{c}}=520 \mathrm{~V}$, and $V_{\mathrm{mod}}=360 \mathrm{~V}_{0 \mathrm{p}}$. The amplitude of $V_{\mathrm{dec}}$ was varied and characteristics of $I_{\mathrm{c}}\left(V_{\text {ret }}\right)$ and energy distributions $f(E)$ were obtained. As for the phase of $V_{\mathrm{dec}}, 8$ kinds of different phases from that of $V_{\text {mod }}$ was applied for each amplitude. The deceleration efficiency explained later was calculated for 8 cases, and the case with the largest deceleration efficiency was taken for evaluation.

In Fig. 5, $f(E)$ of decelerated beam are shown by red curves. Here, (a) and (b) are for $V_{\mathrm{dec}}=100 \mathrm{~V}_{0 \mathrm{p}}$ and $230 \mathrm{~V}_{0 \mathrm{p}}$, respectively, where the values of amplitude are those on $\mathrm{D}_{4}$ or $\mathrm{D}_{8}$. In the figure, $f(E)$ of the incident beam, that is, $V_{\text {mod }}=V_{\text {dec }}=0 \mathrm{~V}_{0 \mathrm{p}}$ is also shown by black curves. As for the incident beam, the value of $f(E)$ is relatively large, so it is scaled by $1 / 3$ in the figure. According to Fig. $5(\mathrm{a}), f(E)$ on $V_{\text {dec }}$ application spreads into both high and low energy regions compared with that of the incident beam. The particles in low energy region are more than those in high energy region, thus the averaged energy decreases, that means the beam is decelerated by the application of $V_{\mathrm{dec}}$. In Fig. 5 (b), it is found that energy decrease, and thus deceleration is enhanced.

The deceleration was quantitatively evaluated. The average energy of the particles can be defined by $\langle E\rangle=$ $\int_{0}^{\infty} E f(E) \mathrm{d} E / \int_{0}^{\infty} f(E) \mathrm{d} E$. Deceleration efficiency can be found by $\eta=\left(\left\langle E_{0}\right\rangle-\langle E\rangle\right) /\left\langle E_{0}\right\rangle$ where $\left\langle E_{0}\right\rangle$ means the average energy of the particles when the modulator and the decelerator are not operated.

Figure 6 shows $\eta$ versus $V_{\mathrm{dec}}$. The deceleration efficiency increases as $V_{\text {dec }}$ increases. This is because number of trapped particles increases as $V_{\text {dec }}$ increases. In the case of $V_{\mathrm{dec}}=230 \mathrm{~V}_{0 \mathrm{p}}$, deceleration efficiency of $32 \%$ was achieved which is the highest record in simulation experiments.

Deceleration effects are also examined by numerical calculation. The calculation is a one-dimensional particle orbital calculation without space charge effect, which is explained in the previous paper [7]. Under the same con- 


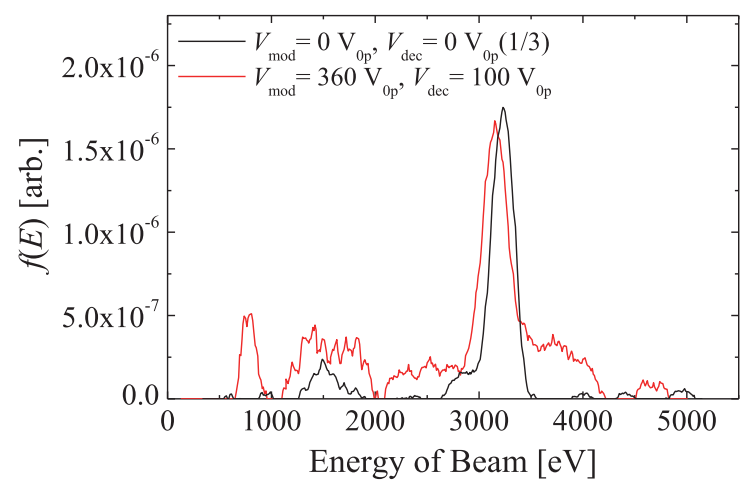

(a) $V_{\mathrm{dec}}=100 \mathrm{~V}_{0 \mathrm{p}}$

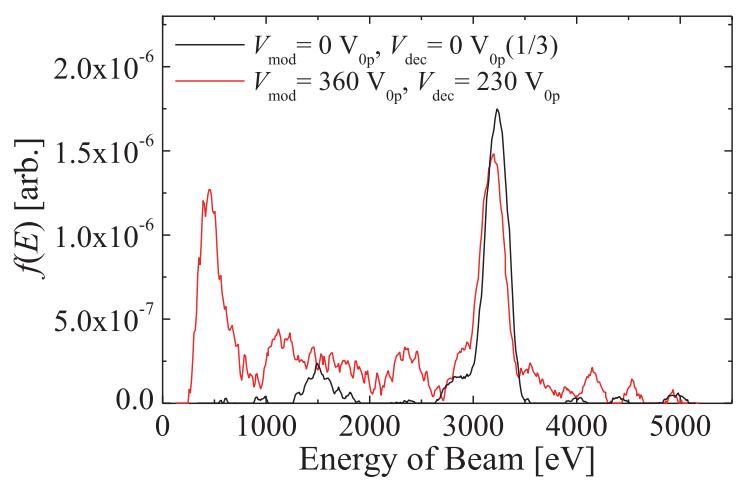

(b) $V_{\text {dec }}=230 \mathrm{~V}_{0 \mathrm{p}}$

Fig. 5 Energy distribution of the decelerated beam.

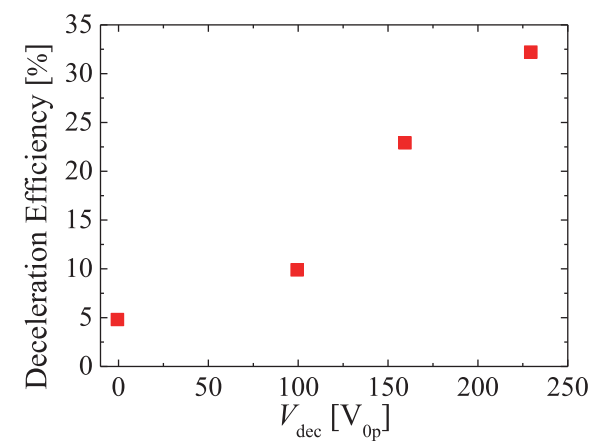

Fig. 6 Deceleration efficiency versus deceleration voltage.

dition with the experiment of $V_{\mathrm{dec}}=230 \mathrm{~V}_{0 \mathrm{p}}$, variation of energy distribution function was examined.

Figure 7 shows energy distributions of the incident and decelerated beams (black and red curves, respectively). The figure can be compared with Fig. 5 (b) directly. Both distribution functions of the decelerated beam are similar except around the energy of the incident beam (3.2 keV). In Fig. 5 (b), there exists significant amount of the component of the incident beam in the decelerated beam while it is quite small in Fig. 7. In the experiment, modulation effect is not enough as in the previous report [3], thus the components of low modulation effect have poor interaction with the deceleration field. As a result,

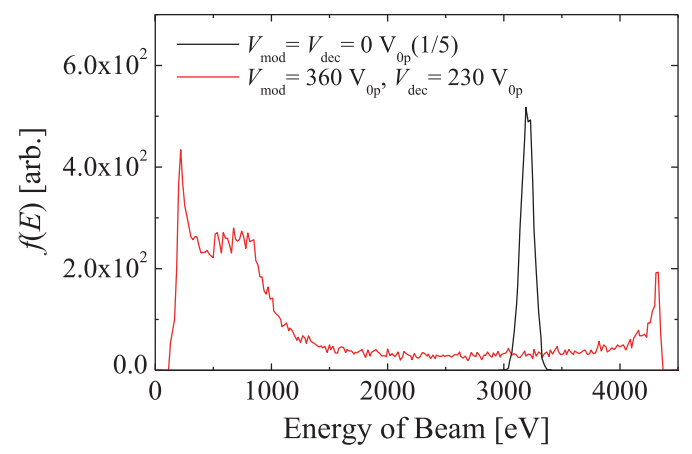

Fig. 7 Calculated energy distribution.

the component of the incident beam remained in the decelerated beam. If the modulation and the deceleration are enough as in Fig. 7, the achieved efficiency will be $55 \%$ within the one-dimensional simulation.

\section{Conclusion}

In this study, we designed the decelerator of a TWDEC simulator based on the constant deceleration scheme, and performed deceleration experiments. As a result, deceleration efficiency increased as the deceleration voltage increased, which was consistent with the theory of the scheme. The maximum deceleration efficiency is $32 \%$ which is the highest record in simulation experiments. The problem was also clarified that the large amount of incident component which was not affected by modulation and deceleration field remained in the decelerated beam, which was not found in the orbit calculation. This component must be related with the problem on modulation process, thus further experimental study on modulation is necessary.

\section{Acknowledgment}

The authors acknowledge valuable discussions with Drs. J. Miyazawa and I. Katanuma. This work was supported in part by the bilateral coordinated research between Plasma Research Center, University of Tsukuba, National Institute for Fusion Science, and Kobe University (NIFS13KUGM082).

[1] H. Momota, LA-11808-C, Los Alamos Natl. Lab., 8 (1990).

[2] J.H. Scott, J.A. George and A.G. Tarditi, Proc. Nucl. Emerging Tech. for Space 2013, Paper 6756 (2013).

[3] K. Fujita et al., 9th General Scientific Assembly of the Asia Plasma and Fusion Association in 2013, TP-32 (2013).

[4] K. Sugihara et al., J. Korean Phys. Soc. 49, S141 (2006).

[5] H. Takeno et al., Fusion Eng. Des. 83, 1696 (2008).

[6] H. Katayama and K. Sato, J. Plasma Fusion Res. 77, 698 (2001).

[7] H. Takeno et al., Jpn. J. Appl. Phys. 39, 5287 (2000). 